\title{
Los aportes implicados en las experiencias recientes de educación popular de la Región Metropolitana, Chile: desde la subjetividad a la transformación social
}

Contributions involved in the recent experiences of popular education in the Metropolitan Region, Chile: from the subjectivity to the social transformation

\author{
Ignacia Palma Salinas*
}

\section{Resumen}

Durante la última década, Chile ha sido testigo de movilizaciones que han puesto en cuestionamiento la legitimidad de nuestro modelo político y económico, instalando la necesidad de cambios estructurales, así las experiencias democráticas, como las de educación popular, han ido tomando fuerza.

El presente artículo es el resultado de una investigación cualitativa que indaga, desde la perspectiva de educadores y educadoras populares, los aportes de sus experiencias en el contexto actual, es decir, los roles que se le otorgan a la educación popular y las transformaciones que ella realiza para educadores y educadoras, educandos y sus territorios. Los principales hallazgos dan cuenta que estas experiencias repercuten en el ámbito microsocial, en tanto transforman las subjetividades involucradas a través de la búsqueda por construir otra realidad y la generación de comunidades.

\footnotetext{
* Socióloga por la Universidad de Chile. Asistente de investigación del Proyecto Fondecyt 1160303 "Movimiento estudiantil en el Chile post dictadura: un estudio longitudinal en los dominios grupal y macrosocial", Universidad de Chile, ignacia.palma11@gmail.com.
} 


\begin{abstract}
During the last decade, Chile has gone through several protest and social movements that have called into question the legitimacy of our political and economic model, installing the need for structural changes, so that democratic experiences, like those of popular education, have been gaining strength.

This article is the result of a qualitative research that explores, from the perspective of the popular educators speeches, the contributions of their experiences in the current context, the roles that they attribute to popular education and the transformations that this practices performs for educators, students and their territories. The main findings raise that these experiences affect the micro-level, while transforming the subjectivities involved through the persecution of building another reality and creating communities.
\end{abstract}

\title{
Palabras clave
}

Educación popular, subjetividades, empoderamiento social.

\section{Keywords}

Popular education, subjectivities, social empowerment.

\section{Introducción: antecedentes históricos de la educación popular}

La educación popular surge en América Latina posteriormente al hito de la revolución cubana, en un contexto en que el devenir histórico de la región estaba fuertemente marcado por procesos de cambio político y social. Se hace presente así la necesidad de construir un conocimiento propio, acorde a la realidad latinoamericana y en la búsqueda por una transformación radical de la sociedad, asumiendo el problema de la humanización y su carácter ineludible (Freire, 2005). Esta corriente pretende llevar a cabo una práctica que es pedagógica y política en el mismo movimiento, con una intencionalidad liberadora (Ander-Egg, 2009). Surgen así, en la región, diversas prácticas que se desarrollan en conjunto con los sectores populares y que pasan a denominarse educación popular. 
En Chile, la educación popular cobra mayor relevancia política durante la dictadura ya que permitió generar una alternativa de resistencia y de reconstrucción del tejido social, ante las dinámicas represivas del Estado. De modo que se constituyó, tanto en este país como en el resto de la región, en un campo para la acción política y la movilización social. Así, estas experiencias fueron parte de las jornadas de protesta que se llevaron a cabo durante la dictadura, las cuales modificaron el cuadro político dictatorial y prepararon el retorno a la democracia.

Sin embargo y posteriormente, en el proceso de la transición a la democracia dichas jornadas quedaron bajo el escenario del cambio de banda, en un pacto sin el pueblo, convirtiéndola en una "transición enajenada" o expropiada a los movimientos populares chilenos (...), excluyendo al mundo social de los "arreglos" de la transición y, sobre todo, de la participación política" (Garcés, 2012: 23).

Dicho proceso abrió para la educación popular una segunda etapa que implicó una redefinición de la misma y una dificultad para proyectar la experiencia acumulada hasta ese momento. Este periodo se caracterizó por un creciente desinterés de la población en la acción política, fenómeno que no es exclusivo de Chile, sino que también se hizo reconocible en el resto de América Latina. Así, es posible observar un debilitamiento de la relación entre las estructuras políticas y la base social, junto con cambios profundos que dan paso a movimientos sociales heterogéneos y multifacéticos, muy críticos de la clase política, la manipulación y la utilización de los movimientos sociales (Bustos, 1996).

En todo este proceso, relevante fue la limitación práctica que encontró la educación popular cuando el financiamiento a las ONG se detuvo, provocando una carencia de recursos que les impidió continuar como lo venían haciendo. Esto acabó por develar que las experiencias desarrolladas en la dictadura habían generado una gran dependencia económica traída por la época de bonanza que implicaba el contar con financiamiento internacional.

Esta situación concreta y el nuevo contexto terminan por provocar una bifurcación en dos caminos de lo que había sido una educación popular con una apuesta más o menos uniforme hasta ese momento, dando paso a una línea que se reorientó hacia la cooperación con el Estado y a otra que se mantuvo en la construcción de un proyecto social alternativo.

La primera lectura sustentó su giro en el alcance limitado de lo local y la ineficacia de aquello que se había llevado a cabo, junto con una opción más propiamente educativa que llevó a considerar la inserción dentro de las escuelas como una acción necesaria, buscando a partir de dicho espacio explotar las metodologías y técnicas acumuladas por la experiencia previa (Bustos, 1996). El devenir de esta línea consistió en el vaciamiento del contenido político de la educación popular y su reducción a metodologías, vinculándose por ejemplo a la CEPAL con el concepto de "educación ciudadana" (Fauré, 2007). 
Sin embargo, la segunda corriente se sustentó en la apuesta por una profundización democrática en conjunto con los sectores populares, e independiente de la óptica estatal y los espacios institucionalizados (Bustos, 1996). Para estos grupos, de acuerdo con el autor antes nombrado, no se trató solamente de incorporar ciertas metodologías, sino de apostar a un conocimiento crítico y autónomo, generando un cuestionamiento profundo de las bases del modelo de desarrollo imperante. Muchos de ellos fueron críticos del primer camino por considerar que se abandonaba la bandera del cambio social y político, optando por un trabajo que renunciaba a una acción de cara a la gente.

De este modo, el proceso de institucionalización de las metodologías de educación popular la llevaron a:

mostrar signos de debilitamiento, profundo cuestionamiento, descrédito de las acciones político-pedagógicas anteriores (...) Esto nos lleva a pensar que el periodo comprendido entre 1995 y el 2004 corresponde a un espacio de invisibilidad de la EP en el país. Al menos en su vertiente proyectiva transformadora (Fauré, 2007: 36).

Esta pérdida de fuerza y posterior renovación se verá remecida para dicho autor a partir de 2004 con la convocatoria al Encuentro Nacional de Educación Popular, cuya invitación fue abierta a ONG y a organizaciones y movimientos sociales. Para el investigador, este fue el hito fundacional que llevó a la pronunciación de ciertas acciones que venían desarrollándose de modo subterráneo, dando cuenta de nuevos sujetos y nuevas prácticas vinculadas a la educación popular.

Así, desde 2004 en adelante se identifica una resignificación de la educación popular donde educadores y educadoras dan cuenta de dos líneas: la educación popular como forma de vida y como herramienta. Respecto de la primera, se configura la idea de que lo fundamental de las prácticas de educación popular es su sustento ético-valórico, que le da sentido a todas las acciones educativas que se realizan, insertándola así en la cotidianeidad donde se despliegan distintas subjetividades. Esto remueve, de cierto modo, la clásica concepción de la política racional, pues aquí lo afectivo activa y actúa "como oxigenador de nuestro razonamiento político" (Fauré, 2006: 128).

En cuanto a la visión de la educación popular como herramienta o como estrategia, se presenta una cierta continuidad con su par de la dictadura, en tanto educadores y educadoras son principalmente "hacedores" que buscan desarrollar una práctica que permite alcanzar los objetivos de transformación planteados. Se define así, estratégicamente, la creación de espacios de diálogo y de encuentro, poniendo en tensión el alcance de lo local (Fauré, 2006).

De este modo, se destacan dos aspectos definitorios de la educación popular: su carácter concientizador y su carácter liberador. Así, por una parte, será todo aquel 
esfuerzo pedagógico que tenga por objetivo la concientización de la parte oprimida de la población, pretendiendo la emergencia de una conciencia propia de clase herramienta. Por otra parte, como proceso liberador, implica la búsqueda de un sistema social alternativo distinto del capitalismo -forma de vida- (Betto, 1984). La intención es, en definitiva, hacer del pueblo un sujeto de su propia historia hacia la construcción de una nueva sociedad y ello se constituye como el hilo conductor del desarrollo de la educación popular chilena en su corriente alternativa.

\section{Metodología}

El presente artículo es parte de una investigación que indagó en los sentidos políticos que educadores y educadoras populares de la Región Metropolitana les asignan a sus acciones educativas. Dicha región es una de las 15 que dividen políticoadministrativamente al país y, pese a ser la más pequeña en extensión, es la más habitada por encontrarse en ella la cuidad de Santiago, capital de Chile.

La investigación aquí presentada tuvo un enfoque cualitativo, y la técnica de producción de información fue la de entrevistas en profundidad, a las cuales se les realizó un análisis de contenido.

El diseño muestral se definió en torno a dos criterios; el primero, en función del tipo de experiencia en la que educadores y educadoras populares participan; el segundo, en función del tiempo de militancia de educadores y educadoras en sus experiencias, entendiendo que la forma en que ésta se interpreta, y los significados y sentidos que se le atribuyen, puede variar según las vivencias que se hayan tenido. Se realizaron 10 entrevistas en profundidad a sujetos que participaran en experiencias autodefinidas como educación popular y que buscaran para las mismas una consistencia política y pedagógica.

Estas experiencias se definen por ser promovidas por organizaciones que Fauré (2006) denomina de "nuevo orden" y que justamente predominan en el escenario de educadores y educadoras populares que se abordaron. Éstas se caracterizan principalmente por tener militantes jóvenes, principalmente universitarios y recién egresados, ser de participación voluntaria, tener un carácter autogestionado y no tener arraigo institucional -ya sea dentro de programas específicos, ONG, iglesias, etc.

Se definieron tres tipos de experiencias de educación popular:

Experiencias culturales: aquellas iniciativas que ponen el énfasis en lo cultural y lo territorial, entendidos ambos en la búsqueda por la reorganización de la idea de clase y su lugar en la construcción identitaria de quienes participan.

Experiencias de capacitación: aquellas iniciativas que tienen como fin principal entregar herramientas, tanto prácticas como teóricas, que resultan útiles para algún objetivo en particular. 
Experiencias educacionales formales: aquellas iniciativas que se constituyen a partir de ciertas falencias identificadas en el sistema educacional chileno y que pretenden ser una alternativa para los sectores populares supliendo estas deficiencias.

Así, la participación en dichas experiencias fueron las que permitieron la definición de educadores y educadoras populares como tal, entendiendo que son aquellos que se comprometen con el ejercicio de la educación popular.

\section{Aportes que realizan las experiencias en educadores y educadoras}

Sustentado en la idea del diálogo, en los procesos de educación popular, educadores y educadoras no serán solamente aquellos que educan, sino también quien es educado a través del diálogo entre todas y todos los participantes. Lo anterior implica la construcción de relaciones ya no en base a la verticalidad, sino apuntando hacia una valoración horizontal de los saberes (Fals Borda, 2009). Hay en los procesos educativos posiciones que los distintos actores asumen, por lo que es pertinente afirmar que educadores, educadoras y educandos son diferentes en tanto asumen roles distintos, sin que ello signifique una relación autoritaria ni que dichos roles sean definitivos.

A continuación, se aborda el aporte que les ha significado participar en sus experiencias de educación popular.

Su participación se determina inicialmente por lo que Fauré (2006) define como una búsqueda, de caminos, de alternativas, de construcción, en la medida en que educadores y educadoras se entienden como sujetos inacabados. Así, para ellas y ellos, el primer aporte tiene que ver con que sus experiencias de educación popular se constituyen como alternativa ante la búsqueda de un espacio de organización acorde con sus convicciones políticas, las cuales se sustentan en la idea de que es posible un cambio, construir otra realidad, una sociedad distinta, y encontrarse con otros y otras con los que se comparten estas aspiraciones políticas.

Se parte de una lectura inicial donde la historia se presenta como posibilidad y no como determinación, lo que desata el reconocimiento de las propias capacidades para poder intervenir en el mundo, donde la comprensión del futuro como problema se enmarca en una lectura rebelde.

Una de las cuestiones centrales que tenemos que trabajar es la de convertir las posturas rebeldes en posturas revolucionarias que nos involucran en el proceso radical de transformación del mundo. La rebeldía es un punto de partida indispensable, es el detonante de la ira justa, pero no es suficiente. La rebeldía en cuanto denuncia necesita prolongarse hasta una posición más radical y crítica, la revolucionaria, fundamentalmente anunciadora (Freire, 2008: 75).

Educadores y educadoras populares deciden tomar el camino de una posición anunciadora a través de la participación en experiencias de educación popular, muchas veces teniendo experiencias previas de organización, sobre todo en espacios y colectivos universitarios $y$, aunque en menor medida, en espacios territoriales. 
"Es importante que como trabajadores de la educación tengamos un lugar donde organizarnos (...) porque por lo menos, pa mí, cuando trabajé, trabajaba en el colegio y ahora que trabajo en la $U$, como profe, no hay muchos espacios de organización. Por ejemplo, los espacios sindicales son bien funcionales a los colegios de repente, son bien instrumentales, pa subir el bono o cosas así, pero na, es bien penca. Un espacio de organización de profes, pa mí es como el principal espacio como trabajador de la educación". Militante antiguo, experiencia educacional formal.

"Muchas veces desde la academia, desde el trabajo, por ejemplo, recuerdo mucho en mis años de estudiante universitaria, escribí muchos trabajos con crítica social, crítica al sistema, en todas las clases en verdá, criticaba y cuestionaba lo que estamos viviendo como sociedad, pero... no lo llevaba a la práctica, y yo creo que esta fue una buena forma de hacer algo". Militante antiguo, experiencia cultural.

Se observa que las motivaciones presentes para participar en estos espacios tienen su origen en militancias políticas donde se discuten diversos temas y se desarrollan críticas al sistema, pero sin que esto tenga repercusiones prácticas en un entorno específico o con otros actores. De esta forma, sin abandonar tampoco esos espacios de reflexión, se encuentra en las experiencias de educación popular una vía para "hacer algo" e incidir con acciones concretas sobre aquello que se critica, ante lo cual el trabajo territorial y educativo surge como alternativa. Éste se constituye como principal aporte para educadores y educadoras, en tanto fue buscado por ellos y tiene que ver con el trabajo político que pretenden realizar.

Luego, se reconocen otros aportes que se van generando en la medida en que se participa en las experiencias. Éstos no eran esperados por educadores y educadoras y, sin embargo, lo valoran por lo que implica para sus vidas y proyectos personales.

Así, definen que su participación en estas experiencias cambia su forma de ver, sentir y pensar el mundo, porque en ellas comparten experiencias de organización, conocen otras realidades, comparten con gente de otras generaciones y se hacen parte de los procesos educativos propios y de otras personas. Esto se valora en tanto muestra otras realidades que antes de participar en las experiencias se veían como lejanas o desconocidas.

En todo este proceso se involucra también lo afectivo, como un componente importante, debido a que se reconoce que se entrega y recibe cariño entre quienes participan de la experiencia.

"Pienso que fue como un regalo que me dio la vida aprender acerca de todo este mundo que existe a parte del mundo que uno conoce cuando sale del colegio, así como el colegio, la universidad, el título, el trabajo, tú pensai: en verdá hay un mundo 
aparte po, hay gente que se está organizando, hay gente que está haciendo cosas, hay gente que no solamente se queda en la crítica a la educación formal y hacia las prácticas autoritarias, hay gente que dedica su vida a tratar de cambiarlo po, entonces encuentro bacán que la escuela por una parte me abrió todo este espectro y por otro lado me ha hecho madurar en términos de mi realización, de mi responsabilidad". Militante nuevo, experiencia de capacitación.

"(...) involuntariamente, así como sin buscarlo, hay también un aporte afectivo así muy bacán, como que... yo nunca esperé tener eso a cambio, y no es porque uno sea el más altruista del mundo ni nada, sino porque tú tení cierto objetivo de lo que querí lograr con tu trabajo y no esperai algo tan pa ti". Militante antiguo, experiencia cultural.

Así, en las distintas experiencias de educación popular se observa que la relación entre los participantes se da sobre la base de vínculos que tienen como centro la generación de comunidad, entendida ésta como "las diversas resultantes de procesos sociales de articulación de sentidos en la acción colectiva, que otorgan una cierta unidad en torno a elementos comunes a las y los sujetos que en dichos procesos se vinculan" (Duarte, 2013: 176). La conformación de la misma se caracteriza por su dinamismo, entendiendo que tanto actores como contextos van cambiando y tienen su especificidad en características culturales, sociales y políticas que les son propias.

Dicha generación de vínculos puede motivar a encuentros y comunicación fuera del ámbito de las experiencias. Delgado (2005) entiende este proceso como la creación de instancias de socialización de vivencias y conocimientos. A éstas les atribuye ser un factor relevante para la movilización y participación en organizaciones sociales. Ello entendiendo que un elemento central para analizar estas organizaciones son las relaciones e interacciones sociales que en ellas se producen, de modo que:

la transcendencia radica en la posibilidad de efectuar intercambio de narrativas personales y de prácticas culturales entre los miembros. En otras palabras, los colectivos sociales fungen como espacio en el cual se escenifican vivencias personales, a partir de la puesta en común de variadas formas de pensar, valorar y sentir la realidad y sus problemáticas concretas (Delgado, 2005: 191).

Por otro lado, se reconocen ciertos aprendizajes prácticos que las experiencias aportan, como herramientas para trabajar con otras personas, metodologías, y sobre todo en términos de organización y ciertas prácticas que son fundamentales para funcionar, como mantener canales de comunicación establecidos, cumplir con las responsabilidades que se asumen, hacer actas de las reuniones y otros saberes.

De esta forma, los aportes que educadores y educadoras populares definen para ellos mismos en función de su participación en las experiencias de educación popular son tres: (a) encuentran un espacio de organización donde materializan sus convicciones 
políticas, (b) un espacio en el que construyen nuevas visiones del mundo a partir del reconocimiento de otras experiencias y realidades, y (c) un espacio donde desarrollan ciertos aprendizajes organizacionales prácticos que pueden llevar también a otros ámbitos de su vida.

Todos estos aportes son valorados por educadores y educadoras populares en la medida en que no necesariamente buscaban obtenerlos. Por esta razón, señalan que la participación en sus experiencias les ha aportado más de lo que esperaban; porque inicialmente todos se plantearon objetivos vinculados a un proyecto político, pero las experiencias de las que han sido parte generaron otras formas de ver el mundo y aprendizajes prácticos que aportan en otros ámbitos de sus vidas y desatan otros procesos personales, como el cuestionamiento de su ejercicio profesional y la autoconfianza.

\section{Aportes que realizan las experiencias en educandos}

Educadores y educadoras identificaron cuáles son para ellos los aportes que realizan las experiencias de educación popular a quienes participan como educandos. Se identifica en primer lugar aquello que se busca enseñar o las herramientas que se quieren entregar en la experiencia educativa, vale decir, la historia y los valores de la propia población, herramientas de comunicación popular, nivelar sus estudios de básica y media, entre otros. Esto ya se considera como un aprendizaje importante o un logro, sobre todo para jóvenes y adultos, en tanto su participación en estas experiencias implica para muchos de ellos sacrificar tiempo libre y con sus familias.

Junto con lo anterior es relevante considerar que:

En diferentes estudios sobre los aportes de la educación popular aparece con reiteración la idea de que les permitió a los participantes ganar confianza para reconocer la manera como en sus vidas se manifiesta el control y el dominio, lo cual les exigió realizar procesos constructores de identidad y de toma de conciencia de una lectura crítica de la realidad (Mejía, 2013: 205).

Según el autor antes citado, lo anterior moldea una subjetividad rebelde para la elaboración de alternativas para la transformación de sus entornos. De este modo, se reconoce que la individuación es un ámbito central a ser trabajado, pues lleva a la búsqueda de una sociedad diferente.

En este sentido, estas experiencias estarían constituyéndose en un espacio de organización para educandos, en la medida en que ellos mismos pueden proponer y tomar decisiones sobre la evolución de las mismas, de modo que las distintas instancias van respondiendo mejor a sus necesidades. Así, los procesos de deliberación colectiva van dejando las lógicas individualistas de lado, en tanto se va construyendo comunidad donde se ponen también lazos afectivos y de amistad, se comparte el propio aprendizaje y se producen espacios de encuentro con otras organizaciones, otros actores y otros vecinos que muchas veces no se conocían. 
"Y pa los cabros, primero pa que terminen sus estudios que es lo que quieren y también po, un espacio de organización po, un espacio como de construcción colectiva, que cachen que no te las tení que rascar solo, que siempre cuando tratai de surgir colectivamente es mucho más fácil y mucho mejor que hacerlo solo, como crear ese bichito de la organización". Militante antiguo, experiencia educacional formal.

"Cualquier cosa para compartir lo llevan, no sé po, si vo no tení cigarros, siempre te van a dar uno, entonces entre ellos se forma un grupo de amigos, que también es bonito, porque también viven el proceso juntos. Si a alguno le empieza a ir mal como que se apoyan, entonces también se logra esa comunidad y no existe una competencia entre ellos". Militante nuevo, experiencia educacional formal.

En segundo lugar, los proyectos de educación popular aportarían a los educandos a que comiencen a desarrollar una mayor libertad de pensar, el generar sujetos críticos, que se cuestionen su propia realidad. Educadores y educadoras observan en los educandos que, luego de un tiempo en la experiencia, comienzan a ver y entender el mundo de otra forma, dando cuenta que, de cierto modo, éstas les muestran otra forma de pensar.

"(...) eso es algo... que se ve y es súper tangible que aporta la escuelita, también que los niños se expresan, se sienten; o sea, son considerados en la escuela como sujetos de derechos, por tanto pueden comentar lo que quieran... no hay una autoridad como punitiva, entonces los niños si bien a veces se pasan; o sea, intentamos hacer actividades y como se sienten tan libres, cuesta manejarlos y seguir las actividades, pero eso es algo que también nosotros hemos sembrado, no queremos niños sumisos y por lo tanto nos cuesta más trabajar con ellos porque si algo no les gusta, lo dicen". Militante antiguo, experiencia cultural.

Lo anterior da paso a la emergencia de una autoconfianza en los participantes, quienes comienzan a sentirse parte de algo y a desarrollar una pertenencia con la experiencia en la que participan. Así educadores y educadoras van observando que están cada vez más empoderados, más dueños de la palabra, y van utilizando con mayor propiedad las distintas instancias de las experiencias, viendo también su entorno de otra forma.

"Todo el tema de lo que es lo afectivo, el sentirse parte de algo, el tema de sacar como mayor personalidad; o sea, por ejemplo yo creo que es indudable, indudable el tema de cómo una persona llega a esta experiencia y después se va, es una persona que tiene características distintas, es otra persona po, que tiene ya otra forma de ver el 
mundo, otra forma de ser en el mundo y otra forma de relacionarse con la gente, que tiene otro tipo de discusiones, tiene una pará distinta". Militante antiguo, experiencia educacional formal.

“(...) desarrollaron la autoestima. Yo, puta, por mí yo les diría, 'da lo mismo el $4^{\circ}$ medio $'$, pero si a ellos les importa a mí también me importa. Entonces, primero tiene esa capacidad de autoestima que también se va desarrollando en otra vía, en que se empiezan a valorar ellos mismos (...) porque ellos no necesitan más escuela, ellos necesitan conocerse, ¿cachai?, valorarse como personas, yo creo que eso sí lo hacemos nosotros, o así permitimos que lo hagan, porque al final lo hacen ellos, pero nosotros les damos las herramientas para que lo hagan". Militante nuevo, experiencia educacional formal.

En el caso de niños y niñas, este sentimiento de pertenencia es fundamental ya que en él también se pondrían en juego las confianzas con educadores y educadoras, que construyen en sus experiencias de educación popular un espacio de acogida y de contención para los educandos, entendiendo que muchas veces sus entornos están muy cargados por la violencia.

"Los chiquillos, en ese lugar, tienen un ambiente que podríamos considerar como relativamente seguro, donde ninguno de nosotros los va a insultar, ninguno de nosotros los va a tratar mal, o los va a no sé, si hacen algo mal, recriminárselo, ¿cachai? Ahí es el lugar donde los chiquillos pueden trabajar, ¿cachai?, jugar y aprender, pero como con los demás y sacarlos un poco del entorno de violencia igual que ven afuera". Militante nuevo, experiencia cultural.

"Además de ser un espacio donde intentamos fomentar el pensamiento crítico, el cuestionamiento, también somos un espacio de pertenencia donde los niños se sienten parte de ahí y les damos también mucho cariño; o sea, hay un lazo emocional, hay un lazo significativo con los niños, nosotros les hacemos cariño a los niños, les damos amor que muchas veces les hace falta, sobre todo a estos niños". Militante antiguo, experiencia cultural.

De lo que se trata entonces es de reencontrarse para autoafirmarse, "para rescatarse como seres humanos que piensan, aman, valoran y actúan, reencontrarse para saberse parte del mundo. Hijos de una historia, hacedores de una cultura" (Mejía, 2013: 165). Este reconocimiento individual en el quehacer inmediato inscrito en un micromundo personal puede entonces llevar el análisis hacia los procesos macrosociales.

Todos los aprendizajes mencionados: (a) el reconocimiento de las experiencias como espacio de organización, (b) el generar sujetos críticos y (c) la autoconfianza, son 
resultado de procesos largos de construcción. Es por ello que no se puede esperar ni ser ansiosos buscando resultados inmediatos, pues esto podría generar cierta frustración en educadores y educadoras que valoran que de a poco los participantes vayan mostrando ciertos indicios de aquello que estas experiencias les aportan, en algunas actitudes cotidianas y en su disposición a participar de forma más colaborativa.

Teniendo en cuenta esto, se identifica que el aporte de las experiencias de educación popular a los educandos está atravesado por dos elementos que lo definen: en primer lugar, aportan al desarrollo de la creatividad y la libertad; y en segundo lugar, aportan al empoderamiento social de ellas y ellos.

En cuanto a la creatividad y la libertad, las experiencias de educación en la escuela tradicional se caracterizan por impedir o inhibir ambas, porque lo que se busca es dominar y domesticar.

Por otro lado, en la educación popular se incentivan ambas actividades, en tanto la creatividad desata procesos desafiantes y permite ser renovada. Ello se vincula con que la gente creativa será inconforme y provocadora, lo que pone también en riesgo la estructura social y propicia la movilización. Por ello, cualquier proceso educativo alternativo tiene que considerar como fundante las capacidades creadoras de los educandos, ya que expresarse libremente implica la liberación del propio yo (Gutiérrez, 1997).

Solamente quienes son auténticamente libres pueden ser a su vez creativos, pues "la expresión creadora es válida no sólo porque lleva implícita la autorrealización del yo y la transformación de la realidad, sino por la relación directa que guarda con la misma adquisición de conocimiento (...) la creatividad es un elemento esencial del proceso educativo" (Gutiérrez, 1997:142). Por eso, en las experiencias de educación popular se busca la libertad y la creatividad abandonando la disciplina, debido a que se entiende que esta es algo externo al individuo que se le impone a través de una acción forzada. Esto se hace evidente sobre todo en los espacios que se abren para pensar y elaborar actividades propias, con una libertad vinculada a la libertad de pensar, invitando a actuar creativamente a través del cuestionamiento de la propia realidad.

En segundo lugar, se define el empoderamiento social como aquel que consiste en aquellos procesos que involucran, dentro de cierta comunidad, el reconocimiento y resignificación de las experiencias personales, a través del encuentro con otras y otros, donde los sujetos se van haciendo dueños de la palabra, al mismo tiempo que revalorizan sus experiencias. Ello desata procesos de mayor autoestima y de autorreconocimiento que se ponen en favor de la construcción de un proyecto colectivo, bajo lógicas de apoyo mutuo y de pertenencia a cierta comunidad. Es la afirmación de varios yo de forma simultánea puesta en juego en proyectos colectivos.

Lo anterior se evidencia en las experiencias de educación popular en momentos en que los educandos comienzan a participar más activamente y a reconocer que sus experiencias personales se han forjado socialmente, al mismo tiempo que se van 
sintiendo más dueños de las experiencias en las que participan y que pueden intervenir en las mismas, en tanto se sienten parte de esa comunidad y tienen la confianza para hacerlo. Son claves ahí el desarrollo de la pertenencia, el compartir experiencias, abrir espacios de diálogo y la valoración de lo colectivo por sobre lo individual.

\section{Aportes que realizan las experiencias en los territorios}

El territorio se entiende como un elemento significativo en las interacciones sociales, ya que es un referente identitario que contiene y organiza determinadas prácticas que ponen en juego lo simbólico y lo físico (Duarte, 2013). Es por ello que el territorio es un componente relevante para las experiencias de educación popular, en tanto ellas son parte de procesos educativos en los que se intervienen espacios.

Pese a que educadores y educadoras identifican que el trabajo territorial es una prioridad dentro de las acciones que realizan, la falta de tiempo muchas veces les impide que éste se pueda llevar a cabo de modo más constante y como un elemento prioritario en las acciones que despliegan. Es por esta razón que para educadoras y educadores los aportes menos visibles que realizan sus experiencias educativas están en el ámbito territorial.

De ahí que exista muchas veces una ambigüedad respecto de los actores que compondrían las comunidades de las experiencias, porque en términos ideales ésta estaría compuesta por educadoras, educadores, educandos y otros actores del territorio. Sin embargo, para éstos últimos no se define el rol que tienen, ya que su participación es diferente y minoritaria en relación al involucramiento que tienen los tres primeros.

De todas formas, hay un reconocimiento de las experiencias en sus territorios; sin embargo, éste suele ser superficial, ya que la gente no participa de forma directa en las experiencias, sino que las conocen, porque han oído hablar de ellas, porque han visto sus actividades. Bajo este conocimiento es que en el entorno de estas experiencias se construye una valoración de las mismas, siendo ésta generalmente positiva, porque se intuye o se sabe que están haciendo algo bueno por la población, en tanto generan espacios de participación y por lo tanto cuentan con una cierta legitimidad en el territorio.

“Por ejemplo del... 9 de marzo, nosotros conmemoramos esa actividad el día sábado 28 y llegamos y nos tomamos la calle acá, y proyectamos un documental, estábamos haciendo en esa y de ahí venía un conversatorio y después íbamos a hacer como una velatón, y en esa estábamos tomando la calle y un auto llega y nos empieza a tocar la bocina pa que nos movamos. Entonces nosotros no pescamos po (...) Tocaba la bocina y de ahí una señora así, pero $X$, una señora de aquí de dos casas más allá dijo: '¡Oiga, señor! Dese la vuelta porque aquí los chiquillos están haciendo una actividad, iya váyase, váyase!' y lo echó po, se tuvo que devolver po, y así nosotros nos dimos cuenta 
de que la señora no estaba participando en la actividad y a lo mejor no sabía ni qué era lo que estábamos haciendo, pero ya con el hecho de que éramos nosotros los que estábamos haciendo la actividad en la calle, ya eso era como legitimidad para defender el espacio". Militante antiguo, experiencia educacional formal.

En este caso anecdótico se torna visible la legitimidad de estas experiencias y su modo de vincularse con el territorio donde, si bien hay resistencias y personas que no se interesan en aquello que se hace, también hay otras que valoran que éstas se desarrollen. De todas formas, en dichas construcciones, lo que predomina es la idea de que las actividades que se realizan son como ir al colegio o son puramente recreativas, quedando invisibilizado o en un segundo plano el carácter político de las mismas.

Por otro lado, las experiencias buscan ser desbordadas por los educandos y que ellos y ellas vayan tomando y generando espacios organizativos en los territorios. De esta forma, se entiende que la experiencia educativa entrega ciertas herramientas y conocimientos que les permitirían a ellos y ellas ser quienes puedan liderar otros procesos, en la aspiración de que los territorios vayan teniendo cada vez más organización popular. Esto se trabaja fundamentalmente en las experiencias con niños, niñas y jóvenes que abordan temáticas vinculadas con la historia del territorio, el rescate de su memoria y el reforzamiento de la pertenencia e identidad territorial.

"Que sean niñas y niños comprometidos, y a futuro jóvenes y adultos como con la construcción de una sociedad más justa, como con la solidaridad, ¿cachai?, como con ese tipo de valores, como con el compromiso y también el tema de la identidad de clase también po, comprometidos como con su población, con una identidad y con nociones históricas de su población". Militante antiguo, experiencia cultural.

Lo anterior da cuenta de un acercamiento a la historia social, en tanto lo que interesa no es un rescate del pasado por el pasado, sino que el rescate de la historicidad "que es precisamente el drama de tener que decidir acciones arriesgando el futuro, e insertando e incorporando en ello el pasado" (Salazar, Garcés y Artaza, 2011: 67). Se rescata entonces el sujeto histórico como aquel que, asumiendo su historicidad, construye sociedad sobre la base de su memoria histórica.

Dicha memoria se sistematiza para ponerse en función de la acción, lo que la convierte en una categoría en la construcción de poder. Así, para las experiencias de educación popular que trabajan con grupos de niños, niñas y jóvenes, el rescate y producción de la memoria de sus poblaciones se convierte en una herramienta que se piensa sería útil para que ellas y ellos sean sujetos comprometidos.

En el mismo sentido de lo anterior, las experiencias de educación popular realizan intervenciones en los territorios con las que se van haciendo visibles y van mostrando el trabajo que llevan a cabo a través del uso y apropiación de espacios públicos. 
Por otro lado, no todas las experiencias de educación popular reconocen tener un trabajo establecido en un territorio como es la experiencia de la Escuela de Comunicación Popular, donde luego de una reflexión colectiva se optó por abandonar el trabajo territorial, buscando nuevos formatos que fuesen más pertinentes para aquello que se quería hacer.

"Nosotros igual planteamos esa discusión con respecto a la pega territorial porque nosotros hacíamos la pega en Lo Hermida po, y llegó un punto en el segundo año que estuvimos ahí, nosotros dijimos, 'hueón no estamos haciendo pega territorial acá, no somos ninguno de nosotros de acá, ninguno de nosotros está presente el tiempo suficiente o es parte de las organizaciones del sector pa decir que esta es una pega territorial', y ahí fue cuando planteamos las discusiones respecto al asistencialismo". Militante antiguo, experiencia de capacitación.

Así se abre la pregunta por el significado de realizar un trabajo territorial y qué es lo que implica, el involucramiento que hay que tener, las redes que se generan, el apropiamiento que existe respecto del mismo, si hay que vivir o no ahí y con qué actores hay que vincularse. Ante ello, es fundamental tener en cuenta que en las experiencias de educación popular suelen haber algunos educadores y educadoras que viven en el territorio, pero la mayoría suele no ser del mismo, lo que genera que muchas veces sean vistos como actores externos que vienen del mundo universitario o profesional, generando una cierta distancia.

Queda entonces abierta la pregunta sobre "lo territorial", tantas veces relevado por la educación popular, pero aún con pocas definiciones prácticas y teóricas en un ámbito que pareciera ser prioritario, pero que sigue siendo una tarea que de a ratos parece poco practicable por la falta de tiempo.

Así se observa que existen en los discursos de educadores y educadoras populares formas diversas de entender lo territorial y de involucramiento en los espacios en los que se trabaja, donde lo que pareciera tener más potencia es el abordaje de la historicidad de los territorios a través de herramientas como el rescate de la memoria, con el fin de activar un presente para la construcción de poder. Mientras que la gran deuda sigue siendo cómo lograr que los educandos desborden estas experiencias generando otros espacios de organización.

\section{Conclusiones}

El discurso de educadores y educadoras populares da cuenta de que, para los participantes, los aportes principales de las experiencias se vinculan con las transformaciones de las subjetividades involucradas, ya que se generan procesos en los que las significaciones que se le otorgan a las experiencias propias, la visiones que se 
construyen del yo mismo y del entorno cambian, generando nuevas formas de ser en el mundo.

En el aporte a educadores y educadoras populares esto se evidencia en que, a través de su participación en estas experiencias, logran ser parte de una organización que les permite poner en práctica sus convicciones políticas personales. Esto les facilita incidir de forma concreta a través de la intervención y encuentro con otras realidades que en algunos casos eran poco conocidas, lo que les genera otras formas de ver, sentir y pensar su entorno. Además de lo anterior, se destaca que su participación en estas experiencias les aporta en términos prácticos, ya que les ha permitido incorporar lógicas organizativas que pueden llevar a otros ámbitos de sus vidas.

Por otro lado, se valora para los educandos que la educación popular genera un espacio donde, además de adquirir ciertas herramientas, se constituye en un lugar de organización. Es así como se producen procesos que, en primer lugar, desatan la creatividad y la libertad, en tanto el compartir con otras y otros les hace cuestionarse su propia realidad, ser sujetos más críticos e ir sintiendo libertad para pensar $y$, en segundo lugar, procesos en los que se desarrolla el empoderamiento social que permite mayor autoestima y autoconfianza.

Respecto del aporte que se realiza en los territorios, si bien se define como tema prioritario, para la mayoría de las experiencias abordadas, este es identificado como el aporte menos visible. En general, las experiencias suelen no tener gran incidencia en este ámbito, quedando en tensión la pregunta sobre lo que implica realizar un trabajo territorial y el desafío de cómo llevarlo a cabo.

Así, estas experiencias se definen fundamentalmente por el potencial que tienen en la materialización de la construcción de otra realidad. Ella le otorga un carácter propio a las experiencias de educación popular y las distingue profundamente de la escuela hegemónica reproductora del orden social.

De este modo, su gran valor es que traen de vuelta lo comunitario $y$, en el mismo movimiento, permiten el desarrollo de un empoderamiento social, en tanto se desatan procesos de mayor autoestima y de autorreconocimiento que se ponen en favor de la construcción de un proyecto colectivo, bajo lógicas de apoyo mutuo y de pertenencia a cierta comunidad. Es la afirmación de varios yo de forma simultánea puesta en juego en proyectos colectivos.

El potencial de estas experiencias está entonces en el nivel de lo microsocial, donde se privilegia el desarrollo de relaciones sujeto-sujeto que humanizan los procesos que se llevan a cabo buscando desalojar las lógicas individualistas que impone el neoliberalismo. Así se desencadena una praxis que pone lo colectivo por sobre lo individual y lo cooperativo por sobre la competencia, reforzando identidades y regenerando el tejido social desde los propios sectores populares y los lugares que habitan. 
De este modo, se observa la configuración de estas experiencias de educación popular como experiencias organizativas de carácter democrático que se proponen realizar transformaciones a través del reconocimiento de sus participantes, donde lo comunitario y el compartir con otros y otras se constituyen en una fuente de socialización política y cuestionamiento de la propia realidad abriendo otros posibles campos de acción que sintonizan con las movilizaciones sociales que se desarrollan actualmente en el país, pero que tienen aún un alcance muy limitado en términos de su magnitud y la intervención que realizan en sus territorios.

\section{Bibliografía}

Ander-Egg, E. (2009). Metodologías de acción social. Buenos Aires: Lumen-Hum.

Betto, F. (1984). La educación en los sectores populares. Educación Y Solidaridad, 1, pp. 6-17.

Bustos, L. (1996). Educación Popular: lo que va de ayer a hoy. Última Década, 4, pp. 1 9.

Delgado, R. (2005). Análisis de los marcos de acción colectiva en organizaciones sociales de mujeres, jóvenes y trabajadores. Disponible en: http://ridum.umanizales.edu.co:8080/xmlui/bitstream/handle/6789/1791/501 DelgadoS Ricardo.pdf? sequence $=1$

Duarte, C. (2013). Acción comunitaria con jóvenes. Desafíos generacionales. Última Década, 39, pp. 169-195.

Fals Borda, O. (2009). Una sociología sentipensante para América Latina. Buenos Aires: CLACSO.

Fauré, D. (2007). El nuevo movimiento de educadores y educadoras populares chilenos: tensiones, proyecto y construcción de poder 1999-2006. Disponible en: http://www.tesis.uchile.cl/tesis/uchile/2007/faure d/html/index-frames.html

Freire, P. (2005). Pedagogía del oprimido. México: Siglo XXI.

Freire, P. (2008). Pedagogía de la autonomía. Saberes necesarios para la práctica educativa. Buenos Aires: LOM.

Freire, P. (2009). Cartas a quién pretende enseñar. Buenos Aires: Siglo XXI.

Garcés, M. (2012). El despertar de la sociedad. Los movimientos sociales en América Latina y Chile. Santiago: LOM.

Gutiérrez, F. (1997). Educación como praxis política. México: Siglo XXI. 
Mejía, M. R. (2013). Educaciones y pedagogías críticas del Sur (cartografías de la educación popular). Chile: Quimantú.

Salazar, G. (2009). Del poder constituyente de asalariados e intelectuales (Chile, siglos XX y XXI). Santiago: LOM.

Salazar, G.; Garcés, M. y Artaza, P. (2011). La historia social: sujeto social e historicidad en la construcción de memoria para la acción. Cuaderno de Trabajo. Memorias, Historia y Derechos Humanos, 1, pp. 67-83. 